Andreas Jansen

\title{
Niedriglohnbeschäftigung in Ost- und Westdeutschland
}

\begin{abstract}
In der Bundesrepublik Deutschland muss ein Niedriglohnsektor nicht erst noch geschaffen werden - er existiert bereits. Unterstellt man einen nach West- $(8,49 €)$ und Ostdeutschland $(6,30 €)$ differenzierten gesetzlichen Mindestlohn, so hätten etwa 13\% der abhängig Erwerbstätigen einen Anspruch darauf. Eine detaillierte Analyse des Niedriglohnsektors zeigt deutlich, dass sowohl persönliche als auch strukturelle Merkmale den Niedriglohnbezug beeinflussen. Neben der individuellen Merkmalsstruktur der Niedriglohnempfänger sind die rückläufige Tarifbindung sowie die abnehmende Tarifverbindlichkeit mögliche Gründe für die Entstehung bzw. Ausweitung des Niedriglohnsektors. Da auch die vorhandenen rechtlichen Instrumente zur Flankierung des Tarifsystems Niedriglöhne nur unzureichend regulieren können, stellt ein gesetzlicher Mindestlohn eine durchaus realistische Option für die BRD dar.
\end{abstract}

\section{Problemstellung: Aufbau eines staatlich geförderten Niedriglohnsektors durch ein Kombi-Lohnmodell}

Zum Abbau der Arbeitslosigkeit plant die große Koalition die Schaffung eines staatlich geförderten Niedriglohnsektors in Deutschland. Gemäß Koalitionsvertrag vom 11.11.2005 soll über ein Kombilohnmodell der Anreiz zur Aufnahme einer niedrig entlohnten Beschäftigung erhöht werden. Im Kern würde es sich dabei um einen staatlichen Zuschuss zu niedrigen Löhnen und Gehältern handeln. Über dieses Vorhaben wird momentan kontrovers diskutiert. Dabei stehen vor allem fiskalische sowie beschäftigungspolitische Aspekte im Vordergrund der Debatte. Bei der Idee des Kombilohnes wird vor allem ein entscheidender Aspekt ausgeblendet: Ein Niedriglohnsektor muss in Deutschland nicht erst geschaffen werden, sondern existiert bereits. Wie frühere Studien gezeigt haben (Bispinck/Kirsch/Schäfer 2003; Strengmann-Kuhn 2003), befindet sich ein nicht unerheblicher Anteil der abhängig Beschäftigten im Niedriglohnsegment. Insofern sollte man weniger über die Schaffung, sondern vielmehr über die Regulierung der bereits bestehenden Niedriglohnbeschäftigung diskutieren. In diesem Sinne wäre ein Alternativkonzept zu einem Kombilohn ein gesetzlicher Mindestlohn. Aufgrund seines allgemeinverbindlichen Charakters könnte ein gesetzlicher Mindestlohn als eine Art staatlich festgesetzte Lohnuntergrenze fungieren und somit nicht Existenz sichernde Niedriglöhne verhindern. Ziel dieses Artikels ist es, die potenzielle Wirksamkeit eines gesetzlichen Mindestlohnes zu untersuchen. Im Rahmen einer eigenen empirischen Analyse auf Basis der Daten des Sozio-Ökonomischen Panels (SOEP) ${ }^{1}$ von 2002 sollen

1 Beim Sozio-Ökonomischen Panel handelt es sich um eine repräsentative Wiederholungsbefragung von Privathaushalten in der Bundesrepublik Deutschland. Im Untersuchungsjahr 2002 wurden insgesamt 23.842 Personen befragt.

Arbeit, Heft 2, Jg 15 (2006), S. 98-113 
Ausmaß und Struktur des Niedriglohnsektors detailliert beschrieben werden. Dabei wird die Existenz eines Mindestlohnsatzes unterstellt, der die Grenze zum Niedriglohnsegment markiert. Die Deskription der Niedriglohnbetroffenheit anhand eines „fiktiven“ Mindestlohnes lässt sich vor allem mit seiner großen Verbreitung innerhalb der Europäischen Union begründen. Von den 25 EU-Mitgliedsstaaten besitzen 18 Länder entsprechende Regelungen. ${ }^{2}$ Im ersten Analyseschritt wird zunächst die Niedriglohnbetroffenheit insgesamt sowie getrennt nach Ost- und Westdeutschland dargestellt. Ziel dabei ist es, einen konkreten Mindestlohnsatz festzusetzen, mit dessen Hilfe im zweiten Analyseschritt die Merkmalsstruktur der Niedriglohnempfänger untersucht werden kann. Abschließend soll ein Erklärungsversuch für die Ausweitung der Niedriglohnbeschäftigung in der BRD unternommen und die derzeitige Diskussion über geeignete Regulierungsmöglichkeiten dargestellt werden.

\section{Selektion der Zielgruppe und Bestimmung der Referenzgröße}

Der erste Schritt der empirischen Analyse besteht in der Berechnung eines Referenzlohns, mit dessen Hilfe das quantitative Ausmaß von Niedriglöhnen in der Bundesrepublik Deutschland aufgezeigt werden soll. Dabei wird als Referenzbasis der Bruttostundenlohn gewählt, da sich ein niedriges Erwerbseinkommen auf Monatsbasis nicht nur durch einen geringen Stundenlohn, sondern auch durch eine kurze Arbeitszeit ergeben kann. Eine gängige Variante den Anteil der Niedriglohnempfänger darzustellen, besteht darin, bestimmte Niedriglohnschwellen in Relation zu einem Durchschnitt (relative Lohnpositionen) zu bestimmen. Ein Niedriglohn liegt dann vor, wenn festgelegte Einkommensschwellen unterschritten werden. Dabei ist anzumerken, dass es sich sowohl bei der Wahl der Grenzwerte als auch bei der Festlegung, welche Beschäftigten bzw. Beschäftigungsformen in die Analyse einbezogen werden, um normative Entscheidungen handelt. Demnach stellt sich bei der Bestimmung eines Referenzlohnes grundsätzlich die Frage, wovon der Durchschnitt berechnet werden soll. Um einen realistischen Referenzlohn zu erhalten, empfiehlt es sich, bestimmte Gruppen am Arbeitsmarkt von der Analyse auszuschließen. Im ersten Schritt werden nur im SOEP befragte Personen im erwerbsfähigen Alter berücksichtigt, das heißt Befragte vom 16. bis zur Vollendung des 65. Lebensjahres. Da durch ein Arbeitseinkommen bzw. durch einen potenziellen Mindestlohn Stundenlöhne realisiert werden sollten, die bei einer Vollzeiterwerbstätigkeit zu einem Existenz sichernden Arbeitseinkommen, zumindest für einen Alleinstehenden/eine Alleinstehende, führen, werden folglich Einkünfte aus Nebentätigkeiten außer Acht gelassen. Da außerdem ausschließlich Einkommen aus abhängiger Beschäftigung durch einen gesetzlichen Mindestlohn reguliert werden kann, gehen nur die abhängig Erwerbstätigen in Voll- und Teilzeit sowie in geringfügiger Beschäftigung in die Berechnung ein, für die diese Tätigkeit die Haupteinkommensquelle darstellt. Ausgeschlossen werden Selbstständige, Personen in Aus- und Weiterbildung, Rentner, Wehr- und Zivildienstleistende, Arbeitslose sowie Personen in $\mathrm{ABM} / \mathrm{SAM}^{3}$ und sonstige nicht Erwerbstätige.

Zudem stellt sich die Frage, wie der Durchschnitt berechnet werden soll. In dieser Arbeit wird ein Arbeitseinkommen von weniger als 60\% des Medianeinkommens als Niedriglohnschwelle gewählt. Dieser Wert findet in der wissenschaftlichen Literatur zunehmend An-

2 Neben der Bundesrepublik Deutschland gibt es in Dänemark, Finnland, Italien, Österreich, Schweden und auf Zypern keine gesetzlichen Mindestlohnregelungen.

3 Arbeitsbeschaffungs- und Strukturanpassungsmaßnahmen 
wendung, da die offizielle Armutsgrenze der EU in gleicher Weise definiert wird. Ein gesetzlicher Mindestlohn, der als Zielsetzung ein Existenz sicherndes Arbeitseinkommen für eine Vollzeit erwerbstätige Person hat, sollte sich entsprechend an diesem Referenzwert orientieren. Methodisch empfiehlt die OECD die Verwendung des Median, da diese Maßzahl zum einen die Wirksamkeit eines potenziellen Mindestlohnes am besten widerspiegelt und zum anderen die internationale Vergleichbarkeit gewährleistet. "Using the median, rather than the mean, in the denominator [durchschnittliches nationales Arbeitseinkommen], is probably a better measure of the potential 'bite' of minimum wages. It also provides a better basis for international comparisons given large differences across countries in the dispersion of wages and earnings" (OECD 1997, 8). Der Median halbiert die Einkommensverteilung, das heißt die Hälfte der abhängig Beschäftigten erhält weniger und die andere Hälfte mehr Einkommen. Der Vorteil des Median ist darin zu sehen, dass er gegenüber Extremwerten in der Einkommensverteilung unempfindlicher als das arithmetische Mittel ist. Die folgende Analyse wird mit gewichteten Daten durchgeführt.

\section{$3 \quad$ Niedriglöhne im Überblick}

Im ersten Schritt wird ein Referenzlohn für Gesamtdeutschland berechnet. Dabei ergibt sich ein arithmetisches Mittel von 14,81 € und ein Median von 13,43 €. Daraus folgt als Schwellenwert von 60\% des Median ein Betrag von 8,06 €. Tabelle 1 zeigt ein erhebliches Ausmaß des Niedriglohnsektors in der BRD. Insgesamt 14,6\% der abhängig beschäftigten Arbeitnehmer/innen beziehen einen Bruttostundenlohn von weniger als 8,06€.

\section{Tab. 1: Verteilung der Bruttostundenlöhne nach 60\% des Median (Gesamtdeutschland)}

\begin{tabular}{|l|r|r|r|r|}
\hline & \multicolumn{1}{|c|}{ Häufigkeit } & \multicolumn{1}{c|}{ Prozent } & \multicolumn{1}{c|}{$\begin{array}{c}\text { Gültige } \\
\text { Prozente }\end{array}$} & $\begin{array}{c}\text { Kumulierte } \\
\text { Prozente }\end{array}$ \\
\hline Unter $60 \%$ des Median & 3473453 & 14,6 & 14,6 & 14,6 \\
\hline Über $60 \%$ des Median & 20337797 & 85,4 & 85,4 & 100,0 \\
\hline
\end{tabular}

Quelle: Eigene Berechnungen auf Basis des SOEP 2002

Da ein gesetzlicher Mindestlohnsatz auf Basis einer vorangegangenen wissenschaftlichen Analyse festgesetzt werden sollte, in deren Rahmen die potenzielle Betroffenheit bei verschiedenen Lohnuntergrenzen untersucht wird, stellt sich die Frage, ob ein einheitlicher Mindestlohnsatz, der auf gesamtdeutschen Durchschnittswerten beruht, dem immer noch starken Lohnniveauunterschied zwischen Ost- und Westdeutschland gerecht werden würde. So liegt das arithmetische Mittel für Westdeutschland mit 15,47 € pro Stunde um 4,00 € höher als in Ostdeutschland (11,47 €). Beim Median ergibt sich ein ähnliches Bild. Dieser beträgt in den alten Bundesländern 14,15€, in den neuen hingegen lediglich 10,51€. Aufgrund dieses großen Niveauunterschiedes erscheint es sinnvoll, einen separaten Referenzlohn für beide Landesteile zu berechnen. Als Vorbild für einen differenzierten Mindestlohn könnte das 1996 
verabschiedete Arbeitnehmerentsendegesetz für das Bauhauptgewerbe fungieren. Dabei existiert für ungelernte sowie für qualifizierte Arbeitnehmer und Arbeitnehmerinnen in den alten und neuen Bundesländern jeweils ein separater Mindestlohnsatz. Im Jahre 2005 betrug die aktuelle Mindestlohnhöhe für ungelernte Arbeitskräfte in Westdeutschland 10,36 €, in Ostdeutschland 8,95 €. Bei qualifizierten Beschäftigten betrug die Differenz zwischen den Stundenlohnsätzen sogar 2,82 € (WSI-Tarifarchiv, Stand 31.12.2004). Für die weitere Analyse wird der Datensatz entsprechend aufgeteilt. Dabei ist das Auswahlkriterium die Lage des Arbeitsplatzes.

Bei Betrachtung von Tabelle 2 wird deutlich, dass sich das quantitative Ausmaß Betroffener in Ost- und Westdeutschland, gemessen anhand verschiedener relativer Lohnpositionen, in Prozentwerten nicht stark voneinander unterscheidet. Große Unterschiede zeigen sich jedoch dann, wenn beide Teile Deutschlands mittels des absoluten Wertes von 7,50€ verglichen werden, der von der Gewerkschaft ver.di in die Diskussion eingebracht wurde.

Tab. 2: Anzahl der Betroffenen in Ost- und Westdeutschland bei getrennt berechneten Schwellenwerten

\begin{tabular}{|l|c|c|}
\hline \multirow{2}{*}{ Weniger als $50 \%$ des arithmetischen Mittels } & \multicolumn{2}{|c|}{ Häufigkeit in Prozent } \\
\cline { 2 - 3 } & Westdeutschland & Ostdeutschland \\
\hline Weniger als $60 \%$ des Median & $10,8(7,74 €)$ & $8,6(5,74 €)$ \\
\hline Weniger als $7,50 € /$ Stunde & $13,8(8,49 €)$ & $13,0(6,30 €)$ \\
\hline
\end{tabular}

Quelle: Eigene Berechnungen auf Basis des SOEP 2002 (In Klammern: Bruttostundenlohn (absolut))

In Westen wären bei diesem Betrag 9,4\% der Befragungspersonen betroffen, im Osten dagegen fast ein Viertel. Hier zeigt sich, dass sich das Lohnniveau in den alten und neuen Bundesländern immer noch stark unterscheidet.

\section{$4 \quad$ Zwei Mindestlohnsätze für die Bundesrepublik Deutschland}

Ein einheitlicher Mindestlohn für die Bundesrepublik Deutschland erscheint aufgrund der unterschiedlichen Lohnniveaus in Ost und West nicht sinnvoll. Zur Verdeutlichung der daraus resultierenden Diskrepanz im Hinblick auf die potenziellen Betroffenraten werden verschiedene Lohnuntergrenzen (50\% des arithmetischen Mittels, 60\% des Median, 2/3 des Median) für Gesamtdeutschland jeweils getrennt nach Ost- und Westdeutschland dargestellt (Tabelle 3). 
Tab. 3: Anteil der Betroffenen in den alten und neuen Bundesländern gemessen an gesamtdeutschen Durchschnittswerten

\begin{tabular}{|l|c|c|}
\hline & West & O st \\
\hline & 9,2 & 23,1 \\
\hline $\begin{array}{l}50 \% \text { des arithmetischen M ittels } \\
(7,41 €)\end{array}$ & 11,8 & 28,9 \\
\hline $60 \%$ des Median $(8,06 €)$ & 16,0 & 37,7 \\
\hline $2 / 3$ des Median $(8,95 €)$ & 9,4 & 24,0 \\
\hline $7,50 €$ & & \\
\hline
\end{tabular}

Quelle: Eigene Berechnungen auf Basis des SOEP 2002

Die Zahl der betroffenen abhängig Erwerbstätigen liegt bei allen berechneten Schwellenwerten im Osten mehr als doppelt so hoch wie im Westen. Würde man zum Beispiel 2/3 des gesamtdeutschen Median als Mindestlohnhöhe wählen, hätten in den neuen Bundesländern 37,7\% der Beschäftigten einen Anspruch auf eine Lohnerhöhung, in den alten Bundesländern wären es dagegen „nur“ $16 \%$. Als möglicher Mindestlohn wird der Wert von 8,49 € in Westund 6,30 € in Ostdeutschland gewählt. ${ }^{4}$ Dies entspricht einer Lohnuntergrenze von 60\% des Median. Die Betroffenenrate liegt jeweils bei circa 13\% (Tabelle 2). Hochgerechnet würden insgesamt mehr als drei Millionen Erwerbstätige von der Einführung eines Mindestlohns in dieser Höhe profitieren. Die Praxis des Arbeitnehmerentsendegesetzes im Bauhauptgewerbe zeigt zudem, dass ein getrennter Mindestlohnsatz auch von Gewerkschaftsseite zwar nicht als wünschenswert aber als vertretbar angesehen wird. Hier liegt die aktuelle Differenz bei 1,41 $€$ für ungelernte Arbeitskräfte und 2,82 € für qualifizierte Beschäftigte (Bispinck/Kirsch/ Schäfer 2003, 234f).

Gemessen in Prozent des Vollzeitmedianeinkommens betragen die für die Bundesrepublik gewählten Mindestlohnhöhen 56,0\% in den alten und 57,3\% in den neuen Bundesländern. Im internationalen Vergleich bedeutet dies den zweiten bzw. den dritten Rang hinter Frankreich, wo der Mindestlohn 62,1\% des nationalen Vollzeitmedianeinkommens beträgt. Die Vereinigten Staaten (33,9\%) und Spanien (29,6\%) weisen dagegen sehr geringe Mindestlöhne in Relation zum Medianeinkommen auf. Demnach ist die Spannweite der gewählten Lohnuntergrenzen im internationalen Vergleich sehr groß (vgl. Tabelle 4).

Innerhalb des Niedriglohnsektors in den alten Bundesländern liegt das arithmetische Mittel der Erwerbseinkommen bei 6,38 €. Die Einführung eines Mindestlohnes von 8,49€ würde eine durchschnittliche prozentuale Lohnsteigerung für die Niedriglohnempfänger von circa 33\% mit sich bringen. In den neuen Bundesländern wäre eine Lohnsteigerung von 25\% vonnöten (bei einem arithmetischen Mittel von 5,04€ und einem potenziellen Mindestlohn von $6,30 €)$.

4 Zur Vereinfachung der tabellarischen Darstellung wird im weiteren Analyseverlauf in Westdeutschland zwischen $>8,49 €$ und $<8,49 €$ unterschieden, wobei der Wert 8,49 € stets dem Niedriglohnsektor zugeordnet wird. Entsprechend wird die Lohnuntergrenze von 6,30 € in Ostdeutschland ebenfalls dem Niedriglohnbereich zugerechnet. 
Tab. 4: Mindestlohnhöhe in \% des (nationalen) Vollzeitmedianeinkommens (Stand 2002)

\begin{tabular}{|l|c|c|}
\hline & $\begin{array}{c}\text { Mindestlohnöhe } \\
\text { (in nationalerwährung) }\end{array}$ & $\begin{array}{c}\text { Mindestlohn in \% des } \\
\text { (nationalen) }\end{array}$ \\
\hline Frankreich & $6,83 €$ & $\mathbf{M}$ edianeinkom ens (Vollzeit) \\
\hline Ostdeutschland & $\mathbf{6 , 3 0 €}$ & $\mathbf{5 6 , 0}$ \\
\hline Westdeutschland & $\mathbf{8 , 4 9} €$ & 50,6 \\
\hline Irland & $6,35 €$ & 47,5 \\
\hline Niederlande & $7,11 €$ & 44,7 \\
\hline Großbritannien & $£ 4,20$ & 33,9 \\
\hline Vereinigte Staaten & $5,15 €$ & 29,6 \\
\hline Spanien & $2,55 €$ & \\
\hline
\end{tabular}

Quelle: Eigene Darstellung, in Anlehnung an Low Pay Commission 2003: S. $253 \mathrm{f}$

\section{$5 \quad$ Merkmale der Niedriglohnempfänger}

Die Merkmalsstruktur der Niedriglohnempfänger unterscheidet sich in Ost- und Westdeutschland nur geringfügig voneinander. Allerdings muss bei einer Reihe von Merkmalen auf die Auswertung der ostdeutschen Stichprobe verzichtet werden, da die Zellhäufigkeiten oftmals nicht die notwendige Mindestfallzahl aufweisen. Insofern werden an dieser Stelle nur die häufigsten Merkmale für Westdeutschland wiedergegeben. Die Tabellen 5 bis 9 beziehen sich somit nur auf die alten Bundesländer. Bei der Darstellung der Ergebnisse werden zudem zwei verschiedene Aspekte der Niedriglohnbetroffenheit beleuchtet. Zum einen die Verteilung innerhalb des Niedriglohnsektors, d.h. wie viel Prozent der Niedriglohnempfänger ein bestimmtes persönliches oder strukturelles Merkmal aufweisen. Zum anderen die Verteilung innerhalb der Personen- oder Merkmalsgruppe, z.B. der Anteil der abhängig erwerbstätigen Frauen, die einen Niedriglohn beziehen. Entsprechend der bereits getroffenen Vorauswahl wird nur noch der Schwellenwert von 60\% des Median zur weiteren Analyse verwendet.

\section{Merkmale Geschlecht und Ausbildung}

Vor allem Frauen sind von Niedriglöhnen betroffen. Tabelle 5 zeigt, dass nahezu drei Viertel $(72,8 \%)$ der Erwerbstätigen in Westdeutschland, die einen Niedriglohn erzielen, weiblich sind. Das Verhältnis zwischen Männern und Frauen im Niedriglohnsektor beträgt somit etwa 1:3. Insgesamt erhalten 21,5\% aller Frauen einen Bruttostundenlohn von weniger als 8,49€. Dieser Wert ist bei den Männern mit 7\% deutlich geringer. 
Tab. 5: Zusammenhang von Niedriglohn und Geschlecht (Westdeutschland)

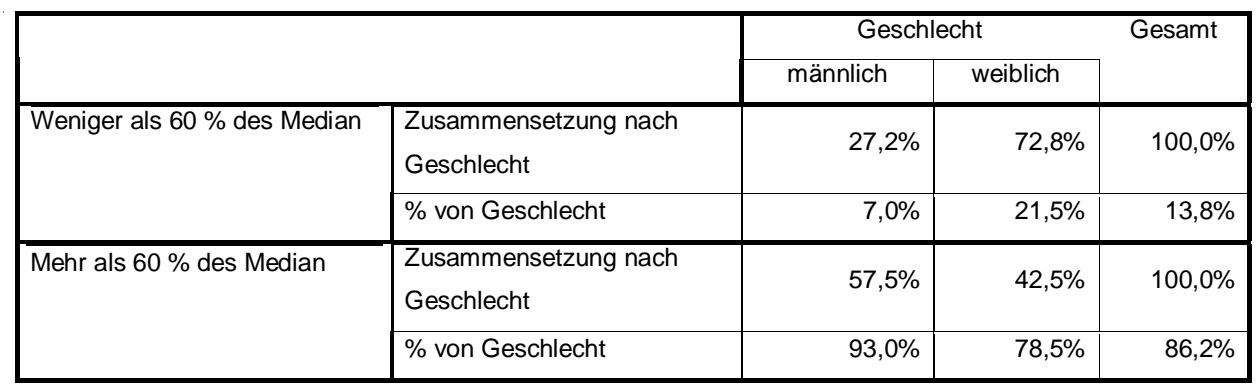

Quelle: Eigene Berechnungen auf Basis des SOEP 2002

Die Analyse der Merkmale Schul- und Berufsausbildung bestätigt den häufig vermuteten Zusammenhang zwischen einer geringen Qualifikation und dem Bezug eines Niedriglohnes, da Erwerbspersonen ohne jegliche schulische oder berufliche Qualifikation mit der größten Wahrscheinlichkeit einen Niedriglohn beziehen. Insgesamt 29\% dieser Personengruppe verdienen einen Bruttostundenlohn von weniger als 8,49 €. Den größten Anteil an allen Niedriglohnempfängern stellen mit 62,7\% hingegen solche Arbeitnehmer, die zwar einen beruflichen Abschluss jedoch kein Abitur haben. „Die große Mehrheit der ArmutslöhnerInnen ist [somit] nicht etwa ,unqualifiziert', sondern weist eine Berufsausbildung auf [...]“ (Schäfer 2003, 425). Dies ist allerdings unter anderem darauf zurückzuführen, dass in der Kategorie „Mit Berufsausbildung jedoch ohne Abitur“" mehrere Merkmalskombinationen aufsummiert worden sind, die zusammen eine sehr hohe Fallzahl ergeben. Entsprechend ist der prozentuale Anteil der Niedriglohnempfänger innerhalb dieser Bildungskategorie mit $14,2 \%$ weniger als halb so groß wie bei den Unqualifizierten, liegt aber dennoch über der durchschnittlichen Niedriglohnbetroffenheit von 13,8\% der abhängig Erwerbstätigen.

\section{Merkmale Alter und Betriebsgröße}

Das Ergebnis der Analyse des Merkmals Alter widerspricht scheinbar der verbreiteten Ansicht, dass vor allem junge Arbeitnehmer von Niedriglöhnen betroffen sind, denn Personen bis zu 25 Jahren tragen lediglich zu 12,9\% zum Umfang des Niedriglohnsektors bei. Mit Ausnahme der 50- bis 55-Jährigen (9,5\%) weisen sämtliche Alterskategorien einen höheren prozentualen Anteil am Niedriglohnbereich auf. Der größte Anteilswert entfällt auf die Altersgruppe der 40- bis 50-Jährigen, denn sie stellen nahezu ein Viertel aller Niedriglohnempfänger (24,6\%). Dieses Ergebnis deckt sich mit der Feststellung von Schäfer, dass der Bezug von geringen Bruttostundenlöhnen kein „Jugendproblem“ darstellt (2003, 425). Allerdings ist die Zusammensetzung durch unterschiedliche Fallzahlen in den verschiedenen Alterskategorien verzerrt. Betrachtet man stattdessen die Verteilung innerhalb der einzelnen Altersgruppen, so zeigt sich eine überdurchschnittlich hohe Betroffenheit der unter 25Jährigen. Mehr als ein Viertel (27,2\%) erhalten einen Lohn, der unterhalb des Schwellenwertes von 8,49 € liegt. Auch bei den 25- bis 30-jährigen Arbeitnehmern ist die Betroffenheit mit $18,7 \%$ noch deutlich höher als die durchschnittliche Niedriglohnbetroffenheit in Westdeutschland (13,8\%).

Die relativ große Diskrepanz zwischen dem Anteil der jungen Arbeitnehmer an allen 
Niedriglohnempfängern auf der einen und der Niedriglohnbetroffenheit innerhalb ihrer Altersgruppe auf der anderen Seite kann u.a. durch längere Ausbildungszeiten und einen damit verbundenen späteren Arbeitsmarkteintritt junger Erwerbspersonen erklärt werden. So befanden sich im Jahre 2002 noch 60\% der 17 - 25-Jährigen in schulischer oder beruflicher Ausbildung (Statistisches Bundesamt 2004, 555). Der geringe Anteil an allen Niedriglohnempfängern resultiert somit hauptsächlich aus der geringen Zahl der unter 25-Jährigen im Datensatz. Mit steigendem Alter sinkt die Wahrscheinlichkeit des Niedriglohnbezuges und erhöht sich erst bei den ältesten Erwerbspersonen wieder deutlich. Knapp ein Fünftel (19,1\%) der über 55-Jährigen bezieht einen unter dem Grenzwert liegenden Bruttostundenlohn. Infolge von Frühverrentungen liegen auch die Erwerbsquoten der älteren Arbeitnehmer deutlich unter denen der 30- bis 50-Jährigen (Statistisches Bundesamt 2004, 99ff). Festzuhalten bleibt aber, dass mit 10\% jede Altersgruppe einen nicht unerheblichen Anteil an Niedriglohnempfängern aufweist.

Es zeigt sich ein deutlicher Zusammenhang zwischen der Betriebsgröße und dem Erhalt eines Niedriglohnes. Mit steigender Beschäftigtenzahl sinkt die Niedriglohnbetroffenheit kontinuierlich. So sind fast 45\% der abhängig Erwerbstätigen in Kleinstbetrieben mit bis zu fünf Beschäftigten von einem Niedriglohn betroffen, in großen Unternehmen mit mehr als 2000 Arbeitnehmern sind es hingegen nur noch etwas mehr als 5\%. Deutlich mehr als die Hälfte der Niedriglohnempfänger (57,1\%) sind in einem Kleinbetrieb mit bis zu 20 Beschäftigten tätig. Zieht man die Definition eines Kleinbetriebes von Bispinck/Kirsch/Schäfer heran, die hierunter Betriebe mit bis zu 99 sozialversicherungspflichtigen Beschäftigten verstehen, so erhöht sich dieser Wert auf nahezu drei Viertel (74,2\%).

\section{Merkmale Branchenzugehörigkeit und Erwerbsstatus}

Die Analyse des Zusammenhanges zwischen dem Bezug eines Niedriglohnes und der Zugehörigkeit zu einer bestimmten Branche auf Basis der Daten des SOEP stellt sich als problematisch dar. So müssen die einzelnen Branchen aufgrund zu geringer Fallzahlen ,[...] zu größeren und teilweise unkonventionellen Gruppen zusammengefasst [...]“ (Schäfer 2003, 426) werden. In den Kategorien „Produzierendes Gewerbe“ und „Sonstige Dienstleistungen“ sind demnach sehr heterogene Branchen enthalten, so dass die Aussagekraft der Ergebnisse für diese Bereiche eingeschränkt ist. Überdies muss eine Analyse des landwirtschaftlichen Sektors unterbleiben, da auch nach der Kategorisierung die Zellhäufigkeiten nicht ausreichen.

Tabelle 6 zeigt, dass der Bezug eines Niedriglohnes nicht ausschließlich an die Zugehörigkeit zu bestimmten Branchen geknüpft ist. Somit bestätigt sich das Ergebnis von Schäfer: „Armutslöhne und ihre BezieherInnen sind auch über die Wirtschaftsbereiche relativ breit gestreut“ (Schäfer 2003, 425). Allerdings scheint die Betroffenheit in manchen Branchen größer zu sein als in anderen. Generell lässt sich zeigen, dass vor allem die Dienstleistungsbranchen einen prozentual höheren Anteil an Niedriglohnempfängern aufweisen. Besonders hoch ist die Betroffenheit in den Bereichen „Freizeitorientierte Dienstleistungen und Gastgewerbe“ mit mehr als einem Drittel, den distributiven Dienstleistungen mit 21,1\% sowie in den Branchen, die überwiegend Dienstleistungen für Unternehmen anbieten mit 15,8\%. Eine unterdurchschnittliche Betroffenheit von Niedriglöhnen lässt sich dagegen im produzierenden Gewerbe (9,6\%) und den öffentlichen bzw. sozialen Dienstleistungen (11,1\%) beobachten. 
Tab. 6: Empfang eines Niedriglohnes in Abhängigkeit von der Branchenzugehörigkeit (Westdeutschland)

\begin{tabular}{|c|c|c|c|c|}
\hline \multirow[b]{2}{*}{ Branchenzugehörigkeit } & & \multicolumn{2}{|c|}{ Einkommensverteilung } & \multirow[t]{2}{*}{ Gesamt } \\
\hline & & $<8,49 €$ & $>8,49 €$ & \\
\hline \multirow[t]{3}{*}{ Produzierendes Gewerbe } & & & & \\
\hline & $\%$ von Branchenzugehörigkeit & $9,6 \%$ & $90,4 \%$ & $100,0 \%$ \\
\hline & $\%$ von Einkommensverteilung & $22,7 \%$ & $34,1 \%$ & $32,5 \%$ \\
\hline \multirow[t]{3}{*}{ Sonstige Dienstleistungen } & & & & \\
\hline & \% von Branchenzugehörigkeit & $14,2 \%$ & $85,8 \%$ & $100,0 \%$ \\
\hline & $\%$ von Einkommensverteilung & $11,1 \%$ & $10,6 \%$ & $10,7 \%$ \\
\hline \multirow[t]{3}{*}{ Distributive Dienstleistungen } & & & & \\
\hline & $\%$ von Branchenzugehörigkeit & $21,1 \%$ & $78,9 \%$ & $100,0 \%$ \\
\hline & $\%$ von Einkommensverteilung & $25,0 \%$ & $14,9 \%$ & $16,3 \%$ \\
\hline \multirow{3}{*}{$\begin{array}{l}\text { Freizeitorientierte } \\
\text { Dienstleistungen und } \\
\text { Gastgewerbe }\end{array}$} & & & & \\
\hline & $\%$ von Branchenzugehörigkeit & $36,5 \%$ & $63,5 \%$ & $100,0 \%$ \\
\hline & $\%$ von Einkommensverteilung & $8,8 \%$ & $2,4 \%$ & $3,3 \%$ \\
\hline \multirow[t]{2}{*}{$\begin{array}{l}\text { Dienstleistungen überwiegend } \\
\text { für Unternehmen }\end{array}$} & $\%$ von Branchenzugehörigkeit & $15,8 \%$ & $84,2 \%$ & $100,0 \%$ \\
\hline & $\%$ von Einkommensverteilung & $8,0 \%$ & $6,8 \%$ & $6,9 \%$ \\
\hline \multirow{3}{*}{$\begin{array}{l}\text { Öffentliche/Soziale } \\
\text { Dienstleistungen }\end{array}$} & & & & \\
\hline & $\%$ von Branchenzugehörigkeit & $11,1 \%$ & $88,9 \%$ & $100,0 \%$ \\
\hline & $\%$ von Einkommensverteilung & $24,5 \%$ & $31,3 \%$ & $30,4 \%$ \\
\hline
\end{tabular}

Quelle: Eigene Berechnungen auf Basis des SOEP 2002

Mit exakt einem Viertel sind die meisten Niedriglohnempfänger im Bereich der distributiven Dienstleistungen beschäftigt. Dies macht noch einmal die hohe Betroffenheit der dazu gehörenden Branchen deutlich, da lediglich 16,7\% aller abhängig Erwerbstätigen in diesem Dienstleistungssektor arbeiten. Den zweitgrößten Anteil am Niedriglohnsektor stellt das produzierende Gewerbe, was sich allerdings durch den hohen Beschäftigtenanteil der sich in dieser Kategorie befindenden Branchen erklären lässt. Insgesamt sind nahezu ein Drittel der hier berücksichtigten abhängig Beschäftigtem in diesem Bereich tätig, ihr Anteil am Niedriglohnsektor mit 22,7\% ist daher unterdurchschnittlich stark. Anders stellt sich das Bild wiederum im Gaststättengewerbe und dem freizeitorientierten Dienstleistungsbereich dar. Obwohl lediglich 3,3\% aller abhängig Erwerbstätigen in diesen Wirtschaftszweigen tätig sind, stellen sie 8,8\% der Niedriglohnempfänger.

Bei der Betrachtung des Erwerbsstatus (Tabelle 7) zeigen sich in Westdeutschland große Unterschiede bezüglich des Anteils der Niedriglohnempfänger. Bei den Vollzeiterwerbs- 
tätigen liegt mit 8,1\% eine unterdurchschnittliche Betroffenheit vor. Dagegen weisen Teilzeitbeschäftigte (23,4\%) und vor allem geringfügig Beschäftigte mit einem Niedriglohnanteil von mehr als drei Viertel eine deutlich größere Betroffenheit auf. Damit stellen geringfügig Beschäftigte mehr als 20\% der Niedriglohnempfänger, obwohl ihr Anteil an den hier berücksichtigten Beschäftigten lediglich 4\% beträgt. Das deutet auf eine erhebliche Lohndiskriminierung dieser Personengruppe hin. Auch der Niedriglohnanteil der Teilzeitbeschäftigten übersteigt mit 32,5\% den entsprechenden Prozentwert in der Grundgesamtheit (19,2\%) deutlich.

Tab. 7: Anteil der Niedriglohnempfänger differenziert nach Erwerbsstatus (Westdeutschland)

\begin{tabular}{|c|c|c|c|c|}
\hline \multirow[b]{2}{*}{ Erwerbsstatus } & & \multicolumn{2}{|c|}{ Einkommensverteilung } & \multirow[b]{2}{*}{ Gesamt } \\
\hline & & $<8,49 €$ & $>8,49 €$ & \\
\hline \multirow{2}{*}{ Vollzeitbeschäftigung } & $\%$ von Erwerbsstatus & $8,1 \%$ & $91,9 \%$ & $100,0 \%$ \\
\hline & $\%$ von Einkommensverteilung & $45,3 \%$ & $81,9 \%$ & $76,8 \%$ \\
\hline \multirow{2}{*}{ Teilzeitbeschäftigung } & $\%$ von Erwerbsstatus & $23,4 \%$ & $76,6 \%$ & $100,0 \%$ \\
\hline & $\%$ von Einkommensverteilung & $32,5 \%$ & $17,0 \%$ & $19,2 \%$ \\
\hline \multirow{2}{*}{ Geringfügig beschäftigt } & $\%$ von Erwerbsstatus & $76,1 \%$ & $23,9 \%$ & $100,0 \%$ \\
\hline & $\%$ von Einkommensverteilung & $22,3 \%$ & $1,1 \%$ & $4,0 \%$ \\
\hline Gesamt & $\%$ der Gesamtzahl & $13,8 \%$ & $86,2 \%$ & $100,0 \%$ \\
\hline
\end{tabular}

Quelle: Eigene Berechnungen auf Basis des SOEP 2002

Zusammenfassend ergibt sich ein sehr heterogenes Bild des Niedriglohnsektors in der Bundesrepublik Deutschland. Dabei scheinen sowohl strukturelle als auch individuelle Merkmale den Niedriglohnbezug zu beeinflussen. Als persönliche Eigenschaft nimmt vor allem das Geschlecht eine herausragende Stellung ein. Allerdings reicht das Merkmal Geschlecht als alleiniger Erklärungsfaktor nicht aus. Vielmehr ist zu vermuten, dass die Kombination von verschiedenen Merkmalen dieWahrscheinlichkeit des Bezuges eines Niedriglohns erhöht. So zeigtbeispielsweise Tabelle 8, dass mit 93\% fast ausschließlich Frauen einer Teilzeit- oder geringfügigen Beschäftigung nachgehen, die ihrerseits eine überdurchschnittliche Niedriglohnwahrscheinlichkeit aufweisen. Erklären lässt sich dies vor allem dadurch, dass für Frauen oftmals ,,[...] Teilzeitarbeit die einzige Möglichkeit [ist], Beruf und Kindererziehung in Einklang zu bringen, d.h. wieder berufstätig zu werden, oder trotz Geburt eines Kindes ihre Berufstätigkeit nicht zu unterbrechen, obwohl mit Teilzeitarbeit erhebliche Benachteiligungen verbunden sind. Denn nach wie vor weist Teilzeitarbeit einen höheren Anteil an gering qualifizierten, belastenden, schlecht bezahlten Tätigkeiten auf als Vollzeitarbeit“ (Bäcker u.a. 2000, 273). 
Tab. 8: Durchschnittliche Bruttostundenlöhne nach Erwerbsstatus und Geschlecht (Westdeutschland)

\begin{tabular}{|c|c|c|c|}
\hline Erwerbsstatus & Geschlecht & $\varnothing$ Bruttostundenlohn in $€$ & $\begin{array}{c}\text { Beobachtete Häufigkeit }^{1} \\
\text { In } 1000\end{array}$ \\
\hline \multirow[t]{3}{*}{ Voll erwerbstätig } & Männlich & 17,59 & 10282 \\
\hline & Weiblich & 14,37 & 4992 \\
\hline & Gesamt & 16,54 & 15274 \\
\hline \multirow[t]{3}{*}{ Teilzeitbeschäftigung } & Männlich & 16,12 & 230 \\
\hline & Weiblich & 12,73 & 3579 \\
\hline & Gesamt & 12,93 & 3809 \\
\hline \multirow[t]{3}{*}{ Geringfügig beschäftigt } & Männlich & 7,27 & 92 \\
\hline & Weiblich & 7,32 & 710 \\
\hline & Gesamt & 7,32 & 803 \\
\hline \multirow[t]{3}{*}{ Gesamt } & Männlich & 17,47 & 10605 \\
\hline & Weiblich & 13,20 & 9282 \\
\hline & Gesamt & 15,47 & 19887 \\
\hline
\end{tabular}

1 Hochgerechnete Daten des Sozio-Ökonomischen Panels.

Quelle: Eigene Berechnungen auf Basis des SOEP 2002

Überdies wird deutlich, dass Vollzeiterwerbstätigkeit noch immer die dominierende Beschäftigungsform auf dem (west-)deutschen Arbeitsmarkt ist. Mit einem Anteil von mehr als drei Vierteln stellen Vollzeiterwerbstätige die weitaus größte Gruppe. Es bestätigt sich, dass Frauen zu einem weitaus geringeren Prozentsatz (53,8\%) einer Vollzeiterwerbstätigkeit nachgehen als Männer (97,0\%). Darüber hinaus werden nochmals sowohl die geschlechtsspezifischen als auch die durch den Erwerbsstatus hervorgerufenen Lohndiskriminierungen sichtbar. Zum einen liegen, mit Ausnahme der geringfügig Beschäftigten, die durchschnittlichen Stundenentgelte der Männer deutlich über denen der Frauen. Dabei ist allerdings zu beachten, dass die geringfügig beschäftigten Männer in der Stichprobe nur einen sehr geringen Anteil an allen geringfügig Beschäftigten stellen. Zum anderen liegen die durchschnittlichen Bruttostundenlöhne bei Teilzeit- und geringfügiger Beschäftigung unter den mittleren Vollzeitstundenlöhnen. Besonders hervorzuheben ist das extrem niedrige durchschnittliche Lohn- 
niveau der geringfügig Beschäftigten. Mit 7,32 € beträgt ihr Durchschnittsstundenlohn lediglich etwa 44,3\% des entsprechenden Vollzeitdurchschnitts.

Zum Abschluss der empirischen Analyse sollen verschiedene Bruttostundenlöhne in den einzelnen Branchen differenziert nach dem Erwerbstatus dargestellt werden (Tabelle 9). Zum einen zeigt sich wiederholt, dass in allen Branchen Vollzeiterwerbstätige zum Teil erheblich höhere Bruttostundenlöhne erzielen als Beschäftigte in atypischen Beschäftigungsverhältnissen. Zum anderen wird deutlich, dass die Stundenentgelte im Branchenvergleich stark variieren.

Tab. 9: Durchschnittliche Bruttostundenlöhne in verschiedenen Branchen in Abhägigkeit vom Erwerbsstatus (Westdeutschland)

\begin{tabular}{|c|c|c|c|}
\hline Branchenzugehörigkeit & Erwerbsstatus & $\begin{array}{l}\text { Mittelwert } \\
\text { in } €\end{array}$ & $\begin{array}{c}\text { Anteil von Teilzeit- und } \\
\text { geringfügiger Beschäftigung }\end{array}$ \\
\hline \multirow[t]{4}{*}{ Produzierendes Gewerbe } & Voll erwerbstätig & 16,65 & \multirow{4}{*}{$10,22 \%$} \\
\hline & Teilzeitbeschäftigung & 12,50 & \\
\hline & Geringfügig beschäftigt & 6,23 & \\
\hline & Gesamt & 16,12 & \\
\hline \multirow[t]{4}{*}{ Sonstige Dienstleistungen } & Voll erwerbstätig & 18,20 & \multirow{4}{*}{$22,13 \%$} \\
\hline & Teilzeitbeschäftigung & 13,36 & \\
\hline & Geringfügig beschäftigt & 6,40 & \\
\hline & Gesamt & 16,92 & \\
\hline \multirow{4}{*}{$\begin{array}{l}\text { Distributive } \\
\text { Dienstleistungen }\end{array}$} & Voll erwerbstätig & 13,79 & \multirow{4}{*}{$31,31 \%$} \\
\hline & Teilzeitbeschäftigung & 11,29 & \\
\hline & Geringfügig beschäftigt & 7,39 & \\
\hline & Gesamt & 12,73 & \\
\hline \multirow{4}{*}{$\begin{array}{l}\text { Freizeitorientierte } \\
\text { Dienstleistungen }\end{array}$} & Voll erwerbstätig & 12,18 & \multirow{4}{*}{$37,99 \%$} \\
\hline & Teilzeitbeschäftigung & 9,25 & \\
\hline & Geringfügig beschäftigt & 6,58 & \\
\hline & Gesamt & 10,79 & \\
\hline \multirow{4}{*}{$\begin{array}{l}\text { Dienstleistungen } \\
\text { überwiegend für } \\
\text { Unternehmen }\end{array}$} & Voll erwerbstätig & 20,31 & \multirow{4}{*}{$27,49 \%$} \\
\hline & Teilzeitbeschäftigung & 12,34 & \\
\hline & Geringfügig beschäftigt & 8,23 & \\
\hline & Gesamt & 17,81 & \\
\hline \multirow{4}{*}{$\begin{array}{l}\text { Öffentliche/Soziale } \\
\text { Dienstleistungen }\end{array}$} & Voll erwerbstätig & 16,92 & \multirow{4}{*}{$31,23 \%$} \\
\hline & Teilzeitbeschäftigung & 14,15 & \\
\hline & Geringfügig beschäftigt & 7,95 & \\
\hline & Gesamt & 15,83 & \\
\hline
\end{tabular}

Quelle: Eigene Berechnungen auf Basis des SOEP 2002

Vor allem die Branchen mit der höchsten Niedriglohnwahrscheinlichkeit, die distributiven sowie die freizeitorientierten Dienstleistungen, weisen geringe mittlere Bruttostundenlöhne 
auf. Darüber hinaus zeichnen sich diese beiden Wirtschaftszweige durch einen hohen Anteil an Teilzeit- bzw. geringfügiger Beschäftigung aus, was als ein möglicher Erklärungsfaktor für das geringe Lohnniveau in diesen Branchen angesehen werden kann. Allerdings können damit nicht die vergleichsweise geringen Löhne von Vollzeiterwerbstätigen erklärt werden, die ebenfalls unterdurchschnittlich sind. Mit 13,79 € (Distributive Dienstleistungen) bzw. 12,18 $€$ (Freizeitorientierte Dienstleistungen) liegen die durchschnittlichen Vollzeitstundenlöhne erheblich unter dem Gesamtdurchschnitt aller Vollzeiterwerbstätigen von 16,54 €. Somit ist zu vermuten, dass auch die Branche für das insgesamt niedrigere Lohnniveau mitverantwortlich ist.

Generell lässt sich festhalten, dass eine Vielzahl von Merkmalen und Merkmalskombinationen die Wahrscheinlichkeit des Bezuges eines Niedriglohns erhöhen. Auch wenn bestimmte Personengruppen eine höhere Wahrscheinlichkeit aufweisen, ist keine Gruppe „frei“ von Niedriglöhnen. Neben persönlichen Eigenschaften scheinen vor allem strukturelle Merkmale wie beispielsweise die Betriebsgröße auf die Lohnhöhe einzuwirken, die durch den Arbeitnehmer nur sehr bedingt beeinflussbar sind. Insofern erscheint ein gesetzlicher Mindestlohn für die Bundesrepublik Deutschland als durchaus diskussionswürdig, da eine erhebliche Anzahl von abhängig Beschäftigten davon profitieren würde.

\section{Erosion des deutschen Tarifsystems}

Abschließend soll die Frage thematisiert werden, wie es im vermeintlichen Hochlohnland Deutschland zur Ausbildung eines Niedriglohnsektors kommen kann. Neben der individuellen Merkmalsstruktur der Niedriglohnempfänger ist eine weitere Erklärung für die Zunahme des Anteils der Niedriglöhne in der abnehmenden Tarifbindung zu sehen. Der Grad der Tarifbindung wirkt sich auf die Gestaltungsfähigkeit und Durchsetzungskraft der Tarifparteien aus. Dieser ist im Zeitverlauf rückläufig. Im Jahre 2003 waren nur noch 46\% der westdeutschen Unternehmen formal tarifvertraglich gebunden, bei den Beschäftigten ist im gleichen Zeitraum ein Rückgang bis auf $70 \%$ zu beobachten. In den neuen Bundesländern ist die Entwicklung im Zeitverlauf ähnlich, das Niveau der Tarifbindung jedoch deutlich niedriger. So lag 2003 der Anteil der tarifgebundenen Unternehmen bei 26\% und somit deutlich unter dem westdeutschen Niveau. Entsprechend geringer war auch der Anteil der Beschäftigten mit 54\%. Noch deutlicher wird der Unterschied zwischen Ost- und Westdeutschland, wenn - an Stelle der gesamtwirtschaftlichen - die Tarifbindung auf Branchenebene betrachtet wird. In sieben Branchen ${ }^{5}$ in den neuen Bundesländern sind weniger als 50\% der abhängig Beschäftigten tarifvertraglich gebunden. In Westdeutschland weist dagegen nur der Wirtschaftszweig der unternehmensbezogenen Dienstleistungen (34\%) eine Tarifbindung von weniger als 50\% auf (Ellguth/Kohaut 2004, 451).

Die abnehmende Tarifverbindlichkeit stellt die zweite Entwicklungstendenz dar. Dabei müssen zwei Erscheinungsformen unterschieden werden. Zum einen können Probleme bei der Anwendung bzw. der Einhaltung geltender Tarifstandards auftreten (Tariftreue), zum anderen kann die vermehrte Anwendung tariflicher Öffnungsklauseln (Flexibilisierung) die Verbindlichkeit von Flächentarifverträgen reduzieren (Bispinck/Kirsch/Schäfer 2003, 40ff). Während die abnehmende Tarifverbindlichkeit zu einer verstärkten Diskrepanz zwischen Tarif-

5 Landwirtschaft (25\%), Investitionsgüterbranche (43\%), Verbrauchsgüterbranche (49\%), Baugewerbe (46\%), Handel und Reparatur (42\%) sowie Organisationen ohne Erwerbszweck (48\%) 
und Effektivlohn führt, wirkt sich eine rückläufige Tarifbindung auf das Niveau der Tariflöhne aus. In einer Reihe von Branchen und Tätigkeiten werden tarifliche Niedriglöhne gezahlt. Besonders betroffen sind die abhängig Beschäftigten in Dienstleistungsbranchen wie dem Einzelhandel (6,71 €) oder dem Friseur- (4,93 €) und Floristikgewerbe (5,94 €) sowie in unternehmensnahen Dienstleistungen wie dem Gebäudereinigerhandwerk (6,36 €) und dem Wach- und Kontrollgewerbe (4,75€) mit tariflich vereinbarten Bruttostundenlöhnen von zum Teil deutlich weniger als 7,00 € (WSI Tarifarchiv (Stand Mai 2005, zitiert nach WSIPressemitteilung vom 11.5.05). Einschränkend muss an dieser Stelle bemerkt werden, dass die Ursache für diese niedrigen Tariflöhne nicht allein in der abnehmenden Tarifbindung zu sehen ist. Vielmehr resultiert dieses zum Teil sehr niedrige Lohnniveau in den untersten Tarifgruppen aus einem Bündel diverser Einflussfaktoren, wie beispielsweise einer ungünstigen wirtschaftlichen Entwicklung innerhalb der jeweiligen Branchen, ein hohes und somit lohndrückendes Angebot an potenziellen Arbeitskräften sowie ein zu geringes Durchsetzungsvermögen der Gewerkschaften aufgrund einer geringen und weiter rückläufigen Tarifbindung.

Neben dem tariflichen Lohnfindungsprozess existieren in der BRD gesetzliche Regulierungsmöglichkeiten, um die abhängig Beschäftigten vor Niedriglöhnen zu schützen. Das derzeit (noch) bedeutendste rechtliche Instrument stellt die Möglichkeit zur Allgemeinverbindlicherklärung vereinbarter Flächentarifverträge dar. Im Rahmen dieses Prozesses können tarifvertraglich vereinbarte Mindeststandards auf nicht tarifgebundene Unternehmen in der jeweiligen Branche ausgeweitet werden. Dabei müssen folgende Bedingungen erfüllt sein:

1. Arbeitgeberverband und Gewerkschaft der jeweiligen Branche müssen sich auf einen Flächentarifvertrag bzw. im Sinne des Arbeitnehmerentsendegesetzes auf einen Mindestlohn-Tarifvertrag verständigen.

2. Die Allgemeinverbindlicherklärung muss von einer beteiligten Tarifpartei beantragt werden.

3. Ein zu gleichen Teilen mit Vertretern der Spitzenorganisationen der Gewerkschaften und Arbeitgeberverbände besetzter Tarifausschuss muss dem Antrag mehrheitlich zustimmen.

4. Die tarifgebundenen Unternehmen müssen mindestens $50 \%$ der im jeweiligen Tarifbereich tätigen Arbeitnehmer beschäftigen.

5. Die Allgemeinverbindlicherklärung muss im „öffentlichen Interesse“ liegen.

Die angeführten Bedingungen müssen prinzipiell in ihrer Gesamtheit erfüllt sein. Allerdings kann von den unter Punkt drei und vier aufgeführten Voraussetzungen abgewichen werden, wenn die AVG zur Behebung eines sozialen Notstandes erforderlich erscheint. Das Instrument der Allgemeinverbindlicherklärung befindet sich ebenfalls in einer Krise. Die Zahl der für allgemeinverbindlich erklärten Flächentarifverträge ist im Zeitverlauf stark rückläufig. Demnach stellt sich die Frage, ob und in welcher Form das Rechtsinstrument der Allgemeinverbindlichkeit reformiert werden kann. Dabei sind folgende Reformmöglichkeiten identifiziert worden (Peter/Kempen/Zachert 2003, 6ff):

1. Die Möglichkeit eines Unterschreitens des $50 \%$-Quorums

2. Verstärkte Anwendung der Klausel „Öffentliches Interesse“

3. Verstärkte und vereinfachte Anwendung der Klausel „Sozialer Notstand“

4. Die Einschränkung des Vetorechts der Spitzenverbände der Tarifparteien 


\section{$7 \quad$ Gesetzliche Mindestlöhne in der Diskussion}

Zur Regulierung von Niedriglöhnen wurde 2005 von der rot-grünen Regierungskoalition eine Ausweitung des 1996 in der Baubranche und Seeschifffahrtsassistenz eingeführten Arbeitnehmerentsendegesetzes beschlossen. Dieses Gesetz basiert auf einer vereinfachten Allgemeinverbindlicherklärung der in der jeweiligen Branche vereinbarten Flächentarifverträge. Dabei kann der Bundesarbeitsminister auf Antrag einer betroffenen Tarifpartei einen Branchentarifvertrag per Rechtsverordnung für allgemeinverbindlich erklären, ohne dass dies der Zustimmung des Tarifausschusses bedarf. Das Vetorecht der Spitzenverbände wurde somit aufgehoben. Auch die weiteren Voraussetzungen wie die Tarifbindung von mindestens 50\% der Arbeitnehmer und das Kriterium „öffentliches Interesse“ müssen bei der gesonderten Allgemeinverbindlicherklärung im Rahmen des Arbeitnehmerentsendegesetzes nicht vorliegen. Die einzig verbliebenen Voraussetzungen sind die Aushandlung eines branchenweit geltenden Mindestlohn-Tarifvertrages sowie die Beantragung der Allgemeinverbindlicherklärung durch eine der betroffenen Tarifparteien.

Ob eine Ausweitung des Arbeitnehmerentsendegesetzes auf andere Branchen die Niedriglohnproblematik wirkungsvoll bekämpfen kann, muss allerdings bezweifelt werden. Eines der Hauptprobleme besteht darin, dass in vielen Wirtschaftszweigen überhaupt kein Flächentarifvertrag existiert, der für allgemeinverbindlich erklärt werden kann. So kommt eine WSIUntersuchung zu dem Ergebnis, dass in gerade einmal sechs von 40 untersuchten Wirtschaftszweigen ein entsprechender Flächentarifvertrag existiert(WSI-Pressemitteilung vom 11.5.05). Dementsprechend würde eine Ausdehnung des Arbeitnehmerentsendegesetzes auf andere Branchen keinerlei Wirkung haben. Überdies ist es fraglich, ob allgemeinverbindliche Mindestlohntarifverträge existenzsichernde Löhne garantieren können. Die derzeit in einigen Branchen beobachtbare Realität von tariflichen Niedriglöhnen lässt in diesem Zusammenhang zumindest Zweifel aufkommen. Insofern ist damit zu rechnen, dass die öffentliche Debatte über die Einführung eines gesetzlichen Mindestlohnes in naher Zukunft nicht abebbt, sondern eher an Schärfe gewinnt.

\section{Literatur}

Bäcker, Gerhard, Reinhard Bispinck, Klaus Hofemann, Gerhard Naegele (2000): Sozialpolitik und soziale Lage in Deutschland. Band 1: Ökonomische Grundlagen, Einkommen, Arbeit und Arbeitsmarkt, Arbeit und Gesundheitsschutz. Wiesbaden. 3., grundlegend überarbeitete und erweiterte Auflage

Bispinck, Reinhard, Johannes Kirsch, Claus Schäfer (2003): Projekt Mindeststandards für Arbeits- und Einkommensbedingungen und Tarifsystem. WSI Projektbericht für das Ministerium für Wirtschaft und Arbeit des Landes Nordrhein-Westfalen (MWA). Düsseldorf

Ellguth, Peter, Susanne Kohaut (2004): Tarifbindung und betriebliche Interessenvertretung: Ergebnisse des IAB-Betriebspanels 2003; in: WSI Mitteilungen 57, 8, 450-454.

OECD (1997): Economics Department Working Papers No. 185. OECD Submission To The UK Low Pay Commission. OECD Online Bookshop. Paris.

Peter, Gabriele, Ernst Otto Kempen, Ulrich Zachert (2003): Rechtliche und rechtspolitische Aspekte der Sicherung von tariflichen Mindeststandards - Rechtsgutachten. WSI Projekt Mindeststandards für Arbeits- und Einkommensbedingungen und Tarifsystem für das Ministerium für Wirtschaft und Arbeit des Landes Nordrhein-Westfalen (MWA). Düsseldorf 
Schäfer, Claus (2003): Effektiv gezahlte Niedriglöhne in Deutschland; in: WSI Mitteilungen 56, 7, 420 $-428$.

Statistisches Bundesamt (Hrsg.) (2004): Datenreport 2004. Zahlen und Fakten über die Bundesrepublik Deutschland. Wiesbaden

Strengmann-Kuhn, Wolfgang (2003): Armut trotz Erwerbstätigkeit. Analysen und sozialpolitische Konsequenzen. Frankfurt/New York

WSI (2005): Untersuchung des WSI. Entsendegesetz: Hohe Hürden in 34 von 40 Wirtschaftsbereichen. Pressemitteilung vom 11.5.2005 (Stand: 21.6.2005)

www.boeckler.de/cps/rde/xchg/SID-3D0AB75D-F3F42E24/hbs/hs.xsl/547_35621.html

Anschrift des Verfassers:

Dipl.-Soz.-Wiss. Andreas Jansen

Universität Duisburg-Essen

FB Gesellschaftswissenschaften

Institut für Soziologie

Soziologie des Sozialstaates - praxisorientierte Sozialwissenschaften

Lehrstuhl Prof. Dr. G. Bäcker

Lotharstr. 65, Raum LF 164

D- 47057 Duisburg

E-Mail: andreasjansen21@gmx.net

Schlagwörter: Arbeitsmarkt, industrielle Beziehungen, national, Personen-/ Berufsgruppen 